\title{
Induced motion of a fixated target: Influence of voluntary eye deviation
}

\author{
THOMAS HECKMANN \\ General Motors Research Laboratories, Warren, Michigan \\ and \\ ROBERT B. POST and LINDA DEERING \\ University of California, Davis, California
}

\begin{abstract}
Induced motion (IM) was observed in a fixated target in the direction opposite to the real motion of a moving background. Relative to a fixation target located straight ahead, IM decreased when fixation was deviated $10^{\circ}$ in the same direction as background motion and increased when fixation was deviated $10^{\circ}$ opposite background motion. These results are consistent with a "nystagmus-suppression" hypothesis for subjective motion of fixated targets: the magnitude of illusory motion is correlated with the amount of voluntary efference required to oppose involuntary eye movements that would occur in the absence of fixation. In addition to the form of IM studied, this explanation applies to autokinesis, apparent concomitant motion, and the oculogyral illusion. Accounts of IM that stress visual capture of vection, afferent mechanisms, egocenter deviations, or phenomenological principles, although they may explain some forms of IM, do not account for the present results.
\end{abstract}

According to a "nystagmus-suppression" hypothesis (Post \& Leibowitz, 1985), illusory motion of a fixated target can arise in an efferent signal for ocular pursuit that opposes involuntary eye movements to maintain a stable fixation. This hypothesis requires two assumptions. First, efferent pathways responsible for ocular pursuit are the same as those that support fixation when stimuli to involuntary eye movements occur. Second, if the first assumption is true, then equivalent sensations of foveated target motion should arise when these efferent pursuit pathways are active, whether or not the target actually moves. Post and Leibowitz (1985) and Heckmann and Post (1988) have reviewed the physiological and behavioral bases for these assumptions in detail.

Corollary to these assumptions, the magnitude of registered target motion should be proportional to the involuntary eye movement that would occur in the absence of fixation. This is supported by work with several illusory motions of fixated targets. First, autokinesis is biased in the direction of eccentric fixation, its vigor proportional to the degree of gaze eccentricity (Adams, 1912; Carr, 1910; Leibowitz, Shupert, Post, \& Dichgans, 1983). This follows from the voluntary effort required to overcome the viscoelastic forces and reflexive tone that tend to retain the eye in the primary position and that increase with eccentricity of gaze. Second, apparent concomitant motion of a fixated target during lateral head excursion (Post \& Leibowitz, 1982) and, third, the magnitude of the oculogyral illusion (Evanoff \& Lackner, 1987) are proportional

Correspondence should be addressed to Thomas Heckmann, General Motors Research Laboratories, 30500 Mound Rd., Warren, MI $48090-9055$. to the amount of vestibuloocular reflex (VOR) that must be suppressed to maintain fixation.

The identical analysis applies to illusory motion of a fixated target opposite the direction of a moving contoured background. In this case, the stimulus to involuntary eye movement is optokinetic. Magnitudes of illusory fixationtarget motion in one direction are correlated with measures of optokinetic nystagmus (OKN) slow phase in the opposite direction across variations in the illumination, velocity (Post, 1986), and duration (Heckmann \& Post, 1988) of unidirectionally moving background contours. This correlation is also obtained across the oscillation frequencies of bidirectionally moving backgrounds (Post, Chi, Heckmann, \& Chaderjian, 1989). Here, the nystagmus-suppression hypothesis assumes that voluntary ocular pursuit is the "fast" component of OKN, which supports a more archaic "slow" OKN to give normal OKN in the absence of a fixation target. When fixation is required, however, pursuit dissociates from archaic OKN and opposes it; in which case, archaic OKN is represented by optokinetic afternystagmus (OKAN; see Heckmann \& Post, 1988; Post, 1986).

The foregoing cases meet the defining criteria (Duncker, 1929) of induced motion (IM). Hence, we also call them IM. However, a number of illusions differing widely in phenomenology and explanation, many of which entail no fixation at all, also fit this definition (for detailed discussion, see Heckmann \& Howard, in press; Heckmann \& Post, 1988; Reinhardt-Rutland, 1988; Wade \& Swanston, 1987).

The use of IM to test the nystagmus-suppression hypothesis has previously depended on correlating IM mag- 


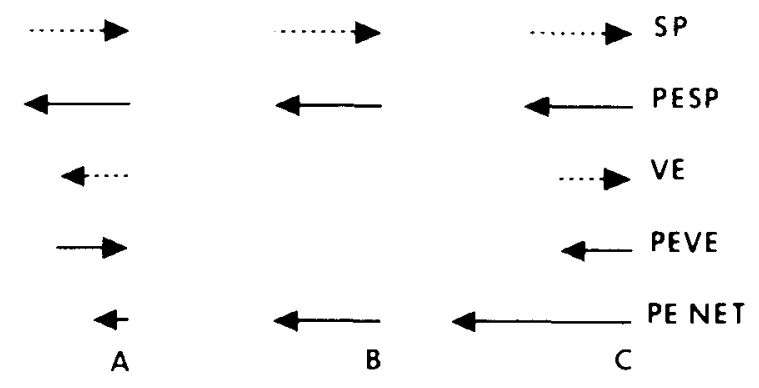

Figure 1. Structure of hypothesis. Dashed arrows represent involuntary factors: the slow-phase (SP) signal in archaic OKN system and the viscoelastic forces and reflexive tone (VE), which tend to abduct deviated eyes back to the primary gaze position. Solid arrows represent the components of pursuit effort, PESP and PEVE, which oppose SP and VE, respectively. Arrows of like type combine algebraically. IM magnitude is proportional to combined pursuit efforts (PE NET). (A) With-stimulus condition, eyes are deviated in the same direction as the OKN slow phase. VE assists in opposing SP, requiring less PESP to oppose the involuntary forces and maintain fixation. In addition, PEVE to maintain deviated gaze is opposite in sign to PESP, so PE NET is less than in: (B) Straightahead condition, eyes are straight ahead. PE NET equals PESP. (C) Opposite-stimulus condition, eyes are deviated opposite the direction of stimulus motion. SP and VE combine positively, requiring positive combination of PESP and PEVE and giving the largest PE NET among the three conditions.

nitude with an oculomotor measure. The present experiment, however, employed the ability of the hypothesis to predict variations in IM magnitude from the fixation task. Fixation of a target located straight ahead gave a baseline of voluntary effort required to oppose the slow phase of OKN, as well as a baseline magnitude of IM. Following the analysis of autokinesis during fixation of an eccentrically deviated target, voluntary effort in the pursuit system is required to oppose viscoelastic forces and reflexive tone in order to maintain fixation. If the deviation is extreme, this effort registers as illusory motion of the fixated target in the same direction as the eye deviation. However, for more moderate deviations, which alone do not reliably produce illusory motion (Adams, 1912; Carr, 1910; Leibowitz et al., 1983), the pursuit efference involved will combine with that needed to oppose the slow phase of OKN, giving different results depending on the direction of the eye deviation with respect to the OKN stimulus, as illustrated in Figure 1.

We predicted that eye deviation in the same direction as the stimulus would result in reduced IM (Figure 1A). Less voluntary efference would be required to oppose OKN slow phase than that in the baseline condition, because OKN slow phase during this eye deviation would also be opposed by viscoelastic forces and reflexive tone, which force the eye back to the primary position. As in autokinesis, these forces require a component of voluntary efference in the same direction as OKN slow phase and voluntary eye deviation, subtracting from the voluntary efference required to oppose OKN. Net voluntary efference and IM, however, would still be in the direc- tion opposite OKN slow phase. This is because the efference required to maintain a moderate gaze deviation is, as noted, too small to give an illusion of motion on its own, compared with the robust illusion of motion that $\alpha c-$ curs during OKN suppression.

For eye deviation opposite the direction of OKN slow phase, we predicted greater IM, because the voluntary efference required to overcome physical and reflexive forces resisting the eye deviation should summate with that necessary to suppress OKN (Figure 1C). Thus, we compared IM magnitudes in three conditions of fixationtarget position, in order of increasing predicted magnitude: eyes deviated in the same direction as stimulus contour motion, eyes straight ahead, and eyes deviated opposite the direction of stimulus motion.

\section{METHOD}

Ten individuals, aged 21 to 37 years, 5 women and 5 men, served as subjects. They were either emmetropic or mildly myopic and wore their normal corrections during the experiment.

The experimental apparatus, the techniques for inducing and measuring IM, and the choice of inducing stimulus parameters have been dealt with in detail elsewhere (Heckmann and Post, 1988; Post \& Heckmann, 1986). All observations were binocular, with the subject seated at the center of a vertical hemicylindrical screen (radius $=50 \mathrm{~cm}$, extending $180^{\circ} \times 95^{\circ}$ ) onto which vertical stripes moving horizontally at $60^{\circ} / \mathrm{sec}$ were projected by means of a shadow caster. Each pair of dark-light stripes subtended $15^{\circ}$. A fixation target approximately $.25^{\circ}$ in diameter was projected onto the screen by means of a laser and a galvanometer-mounted mirror.

The method for measuring IM magnitude, originally employed by Post and Heckmann (1986), was based on reports (e.g., Wallach, Bacon, \& Schulman, 1978) that orthogonal directions of IM and real fixation-target motion combine to yield an apparent path of motion that is the vector sum of the two. During exposure to horizontally moving contours, the subject continuously adjusts a rod manually by feel to match the apparently slanted path of travel resulting from real vertical motion of a fixation target and a horizontal vector of IM. The method is effective in capturing moment-to-moment changes in IM magnitude during prolonged stimulation, permitting the dynamics of the illusion and its aftereffects to be studied (Heckmann \& Howard, in press; Heckmann \& Post, 1988; Post \& Heckmann, 1986; Post \& Lott, 1990). Measurements correlate highly with a technically more complex version of the method in which the horizontal IM vector is canceled rather than matched; errors owing to matching a manually palpated slant to a visual one are negligible compared with differences in magnitudes of $\mathrm{IM}$ obtained across stimulus conditions (Heckmann, 1986). IM of a fixated target observed with this method correlates highly with that measured by apparent horizontal displacement of a fixation target that is stable in the vertical, as well as the horizontal, direction (Post et al., 1989) and is independent of vection (induced motion of the self) experienced during prolonged exposure to large, rapidly moving backgrounds, even though such stimuli are optimal for generating vection as well as robust OKN (Heckmann \& Howard, in press; Heckmann \& Post, 1988).

On each trial, the subject initially sat in darkness and indicated when ready for the trial to start. Each trial began with the appearance of the fixation target and the moving striped background, whereupon the subject began to continuously adjust the slant of the rod to match the apparent slant of the path of the fixation target. The target moved upward $15^{\circ}$ at $3 \% \mathrm{sec}$, was extinguished, immediately reappeared at its original starting point, and began moving upward as before. The subject refixated it and tracked it upward again without interrupting the continuous slant-matching task. The 
extent of the vertical path of the spot bisected the horizontal plane of gaze, so that there was no net deviation of gaze from the horizontal. Trials lasted $30 \mathrm{sec}$, during which slant was sampled every $10 \mathrm{sec}$.

There were three pairs of trials per subject. In one pair of trials, the fixation target was positioned at the subject's median sagittal plane (straight ahead). In another pair of trials, it was positioned $10^{\circ}$ left of straight ahead. In a third pair, it was placed $10^{\circ}$ right of straight ahead. Within each pair of trials, the striped background moved left in one trial and right in the other. The orders of fixationtarget position and background direction were randomized within each subject. This scheme gave three conditions of direction of fixation with respect to the direction of background stimulus motion, with leftward and rightward stimulus motion being represented in each condition. In one condition, target fixation required deviation of the eyes away from straight ahead in the same direction as background stimulus motion (the with-stimulus condition), the head being stabilized in line with the body by means of a dental-impression bitebar so that only the eyes deviated to maintain target fixation. In a second condition, target fixation was straight ahead of the subject (the straight-ahead condition). In the third condition, target fixation required deviation of the eyes away from straight ahead in the direction opposite that of background motion (the opposite-stimulus condition).

Perceived slants were sampled at 10, 20, and $30 \mathrm{sec}$ after trial initiation to permit verification of a previously reported gradual increase in IM magnitude during prolonged stimulation (Heckmann \& Howard, in press; Heckmann \& Post, 1988; Post \& Chaderjian, 1988; Post \& Heckmann, 1986; Post \& Lott, 1990) and evaluation of its interaction with the direction of fixation. Prior to analysis, data were trigonometrically converted to the horizontal IM velocity vectors required to give the reported apparent slants.

\section{RESULTS}

A three-way within-subject analysis of variance assessed three main effects (stimulus duration, fixation direction, and stimulus direction) and their interactions (Table 1). The absence of significant interactions among the main effects allowed each to be considered independently.

Figure 2 represents the effect of stimulus duration. The illusory horizontal velocity of IM, averaged over subjects and collapsed across fixation direction and stimulus direction, is plotted at 10,20 , and $30 \mathrm{sec}$. The gradual increase in IM magnitude with stimulus duration replicates findings with other subject groups (Heckmann \& Howard, in press; Heckmann \& Post, 1988; Post \& Chaderjian, 1988; Post \& Heckmann, 1986; Post \& Lott; 1990). The

Table 1

Analysis of Variance

\begin{tabular}{lrrr}
\hline \multicolumn{1}{c}{ Source } & $d f$ & \multicolumn{1}{c}{$F$} & $p$ \\
\hline Stimulus Duration & 2 & 25.27 & $<.001$ \\
Fixation Direction & 2 & 5.81 & $<.025$ \\
Stimulus Direction & 1 & 10.31 & $<.025$ \\
Stimulus Duration $\times$ Fixation Direction & 4 & 1.07 & n.s. \\
Stimulus Duration $\times$ Stimulus Direction & 2 & 1.35 & n.s. \\
Fixation Direction $\times$ Stimulus Direction & 2 & 1.24 & n.s. \\
Three-Way & 4 & .64 & n.s. \\
\hline
\end{tabular}

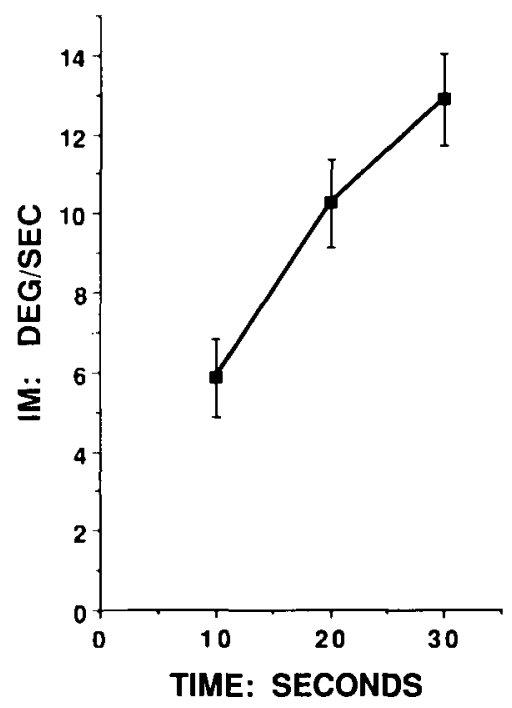

Figure 2. Average over 10 subjects of $\mathrm{IM}$ magnitude sampled 10 , 20, and 30 sec during exposure to hemicylindrical background of projected vertical stripes moving horizontally at $60^{\circ} / \mathrm{sec}$. IM magnitude increases with stimulus duration. Error bars represent $\pm 1 S E M$.

increase in IM magnitude over time was statistically significant $[F(2,18)=25.27, p<.001]$.

Histograms in Figure 3 represent average IM magnitudes for the three conditions of fixation direction, collapsed over the two other factors. The difference in IM magnitude across the three conditions was statistically significant $[F(2,18)=5.81, p<.025]$. Differences across conditions were also in the predicted order: the smallest IM magnitude occurred in the with-stimulus condition, and the greatest occurred in the opposite-stimulus condition. A post hoc comparison between treatments revealed a significant difference between the with-stimulus and opposite-stimulus conditions (Scheffé criterion, $p<.01$ ), but not between other conditions.

There is also a significant effect of stimulus direction $[F(1,9)=10.31, p<.025]$, in which average rightward $\mathrm{IM}$ in response to leftward background motion was $8.2 \%$ and mean leftward IM in response to rightward stimulation was $11.2 \%$ sec. This directional bias is presumed to be an artifact of the manner in which the hand-held rod was mounted on the apparatus. Its plane of rotation was oblique rather than normal to a frontal plane of the subject, approaching the subject from the left to the right. Thus, the wrist of the right hand was already hyperextended toward the right when the rod was gripped without any slant, giving an attenuated range of wrist motion in this direction (in response to leftward stimulation), compared with the range available for leftward wrist rotation (in response to rightward stimulation). Other studies in which the plane of rod rotation was normal to a frontal plane have found no effect of stimulus direction (Heck- 


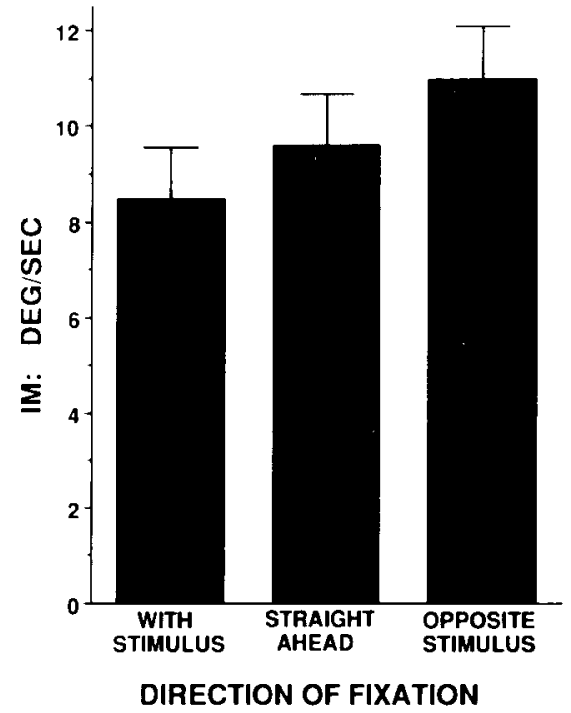

Figure 3. IM magnitude averaged over 10 subjects and three durations for with-stimulus, straight-ahead, and opposite-stimulus conditions. IM magnitude increases across fixation conditions as predicted in Figure 1. Error bars equal \pm 1 SEM.

mann \& Howard, in press; Post \& Lott, 1990). The stimulus-direction effect in the present experiment did not, in any event, influence responses to stimulus duration and fixation direction, as shown by the absence of interactions among the three main effects.

\section{DISCUSSION}

When subjects fixate a horizontally stationary target in the presence of prolonged unidirectional background movement, the magnitude of fixation-target IM decreases when fixation requires deviation of the eyes in the same direction that the slow phase of OKN would otherwise occur. This is consistent with a reduction in the voluntary fixational effort required to suppress the slow phase of OKN. Suppression is partially assisted by viscoelastic and reflexive forces resulting from the eye deviation. Also, a component of voluntary effort, required to maintain eye deviation in the same direction as the slow phase, subtracts from the effort that opposes nystagmus. IM magnitude increases when the eye deviation required to fixate is opposite the direction of the suppressed OKN slow phase. This is consistent with an increase in the voluntary effort to suppress nystagmus, which is required to overcome viscoelastic and reflexive forces resisting the eye deviation.

These results do not conflict with pursuit as the efferent mechanism for suppression of nystagmus by retinally stabilized or imaginary targets (Howard, Giaschi, \& Murasugi, 1989; Wyatt \& Pola, 1984). Evidence for the physiological identity of effector mechanisms in pursuit and nystagmus suppression has already been reviewed (Heckmann \& Post, 1988). Nystagmus suppression oc- curring with afterimages and imaginary targets is consistent with this identity, since pursuit itself is similarly effective with afterimages (Mack \& Bachant, 1969) and during imaginary and predicted target motions (Steinbach, 1976; Whittaker \& Eaholtz, 1982). A predictive component of nystagmus suppression has also been detected (Larsby, Hyden, \& Odqvist, 1984).

The present results argue against an account that considers IM the cause, rather than the result, of pursuit efference (Wyatt \& Pola, 1979). First, the gradual increase in IM magnitude over time is highly correlated with an increase in optokinetic system activity as assessed through OKAN (Heckmann \& Post, 1988). Causal precedence of IM over pursuit would require an IM that builds up over time independently of the duration effect on the optokinetic system. However, no principle available from alternatives to the nystagmus-suppression account independently explains the duration effect on IM and its exquisite tuning to the dynamics of the optokinetic system (Heckmann \& Post, 1988; Post et al., 1989). Since evidence reviewed by Heckmann and Post (1988) indicates that the pursuit signal is the mechanism for nystagmus suppression, it is simpler to assume that nystagmus suppression is causally prior to IM. Similarly, if examination of alternative accounts of IM phenomena supply no basis independent of nystagmus suppression for the present fixation-direction effect, then nystagmus suppression itself remains the sole explanation of this effect. A number of explanations apart from nystagmus suppression have been developed for the wide variety of phenomena defined as IM, and they fall into four main categories: vection-entrained IM, oculocentric IM, object-relative IM, and subject-relative IM. The ability of each to explain the present results is considered, the first two as valid manifestations of semiautonomous motion mechanisms that function within different physiological frames of reference and produce their own characteristic forms of IM.

In vection-entrained IM, stationary contours within or adjacent to a vection-inducing display are often experienced as moving along with the self during vection (Duncker, 1929; Fischer \& Kornmüller, 1930; Warren, 1895). Although use of small contour fields is no guarantee against vection (Johansson, 1977), large fields of contour in prolonged unidirectional motion are optimal not only for vection, but for OKN as well. Their effectiveness in stimulating the optokinetic system has made such fields the stimuli of choice in testing the nystagmussuppression hypothesis through the use of IM. Yet, OKN and vection can be directionally dissociated by placing the OKN stimulus closer to the subject than the vection stimulus (Brandt, Dichgans, \& Buchele, 1974; Brandt, Dichgans, \& Koenig, 1973). Using a similar stimulus configuration, Heckmann and Howard (in press) obtained complete directional dissociation of fixation-target IM measured by the present method not only from vection, but from vection-entrained IM; the two forms of IM could be driven in opposite directions at the same time. Furthermore, measures of vection and fixation-target IM were 
quantitatively independent of one another, regardless of their relative directions, showing that fixation-target IM is unaffected by concurrent vection. The present results, therefore, are not attributable to any change in vectionentrained IM accompanying fixation-target IM.

Heckmann and Howard (in press) interpreted vection and vection-entrained IM as illusory motion occurring in an "exocentric" frame of reference for relations between the world and the whole body. The entire frame of reference for "egocentric" motions of objects relative to the self is carried along with the self during vection. Vectionentrained IM thus adds subjective velocity to objects undergoing egocentric motion; they move "along with" the self, but only as fast as the self is moving. Thus, there is no additional registration of egocentric motion-motion relative to the self-giving no effect on the IM of a fixation target when registered as the horizontal vector of an apparent slant. Egocentric motions and IMs may further be broken down into "headcentric" and "oculocentric" forms.

Headcentric motion is represented by motion sensations associated with ocular pursuit and illusions arising from nystagmus suppression (such as IM in the present study), since these experiences are fundamentally about motion with respect to the head. Oculocentric real motion and IM, which arise from afferent motion mechanisms operating in retinal coordinates, are superimposed on the headcentric form. Oculocentric mechanisms are those responsible for such effects as spatial and velocity tuning of the IM of stationary, as opposed to moving, gratings (Levi \& Schor, 1984) and IM in different parts of the visual field in opposite directions at the same time (Brosgole, 1968; Gogel, 1977; Heckmann \& Howard, in press; Nakayama \& Tyler, 1978). In such cases, the motion sensation is often "fleeting and ephemeral" (Heckmann \& Post, 1988), occurring at full strength immediately upon viewing and fading in and out, in contrast with the robustness and gradual increase in IM of the headcentric or nystagmus-suppression type. Preliminary measures of simultaneous, but opposite, IMs of the oculocentric type made with the present slant-matching method show that this method does indeed register oculocentric IM. However, the magnitude of the oculocentric form is negligible compared with that of the nystagmus-suppression type (Heckmann \& Howard, in press), representing a few minutes of arc per second of subjective velocity. Differences among fixation directions in the present study were measurable in degrees per second rather than minutes per second and were also robust across time, as indicated by the gradual buildup in magnitude and the absence of interaction between stimulus duration and fixation direction. These facts argue against oculocentric IM as the source of the present results.

Another argument against oculocentric factors in the present results is the symmetry of retinal configuration across fixation conditions. No retinal differences were available across conditions that might serve as a basis for oculocentric effects on fixation direction. Symmetry of configuration across fixation conditions also eliminates the object-relative-motion account (Duncker, 1929), because it imputes IM to a change in the position of a stationary target relative to that of a background, such as a moving rectangle, which develops an asymmetrical visual frame of reference as it moves. Stimulus symmetry also eliminates the subject-relative account (Bridgeman \& Klassen, 1983; Brosgole, 1968; Duncker, 1929), in which illusory motion of a stationary target is interpreted from an apparent shift in the visual egocenter within a shifted, asymmetrical frame of reference. Further disconfirmation of this account is found in the fact that a positional shift in the visual egocenter in the direction of background motion develops very slowly, taking about $60 \mathrm{sec}$ to complete (Post \& Heckmann, 1986). However, a slowly developing factor did not influence the effect of fixation direction, as shown by its failure to interact with stimulus duration in the analysis.

The requirements of vection induction, the independence of fixation-target IM from vection, the feebleness of oculocentric IM (relative to that of a single fixation target), the ad hoc nature of phenomenological accounts, and the dynamics of visual egocenter change during induction leave nystagmus suppression alone to account for IM of the classical type: robust, continuous illusory fixation-target motion induced by a moving rectangular frame (e.g., Duncker, 1929; Rock, Auster, Schiffman, \& Wheeler, 1982; Wallach et al., 1978; Wallach \& Becklen, 1983). This idea receives positive support from the identical dependence of OKN and IM on the oscillation frequency of a frame inducer (Post et al., 1989). Also, depth adjacency in IM, originally studied with such stimuli (e.g., Gogel \& Koslow, 1972), can also be attributed to nystagmus suppression. This is because adjacency of convergence to an inducing background determines the direction of IM more strongly than does simple adjacency of fixation target and inducer (Heckmann \& Howard, in press). This is similar to the dependence of OKN on the depth relationship of convergence and inducer (Howard \& Gonzalez, 1987; Howard \& Simpson, 1989).

Even so, it should be clear from the foregoing that the nystagmus-suppression hypothesis is not intended to explain all IM phenomena. Rather, IM of a fixated target has been used as an experimental model to test various implications of nystagmus suppression when the stimulus is optokinetic, versus vestibular or spontaneous, as in other illusions of fixation-target motion.

Certain forms of IM still pose a challenge to the proposed categories of exocentric, headcentric, and oculocentric motion-sensitivity mechanisms. A case in point is IM in depth with respect to a frontoparallel plane (Gogel \& Griffin, 1982). Nystagmus suppression may yet, however, serve as a unifying explanation for other cases that seem at first to defy these categories. For example, the direction and magnitude of $\mathrm{IM}$ is influenced by attention to competing inducing stimuli (Gogel \& MacCracken, 1979; Gogel \& Sharkey, 1989; Gogel \& Tietz, 1976). This is consistent with enhancement of OKN gain by attention directed at a moving display (Barnes \& Hill, 1984; Cheng 
\& Outerbridge, 1975; Dubois \& Collewijn, 1979; HolmJensen, 1984). The fact that IM occurs with discrete, as well as continuous, changes in the background (Bridgeman \& Klassen, 1983) does not argue against nystagmus suppression, since OKN can occur with a stroboscopically illuminated stimulus (Schor, Lakshminarayanan, \& Narayan, 1984). Apparent retrograde motion of a small stationary target when tracking the inducing stimulus has been termed IM (Gogel \& Griffin, 1982; Wallach et al., 1978). Yet, this is also an instance of the Filehne illusion, which can be interpreted as a failure of the position constancy signal associated with ocular pursuit because the need for voluntary pursuit of a large inducer is small, the inducer being an effective stimulus to OKN (Post \& Leibowitz, 1985). A position constancy signal associated with pursuit effort to suppress nystagmus may also explain an underregistration of moving-frame displacement observed during IM (Rock et al., 1982). This signal pushes the subjective visual egocenter opposite the direction of pursuit effort (Post \& Heckmann, 1986), that is, in the same direction as stimulus motion. Displacement between the subjective egocenter and the stimulus is thus less than actual stimulus motion relative to the observer.

\section{REFERENCES}

Adams, H. F. (1912). Autokinetic sensations. Psychological Monographs, 14, 1-45.

Barnes, G. R., Hill, T. (1984). The influence of display characteristics on active pursuit and passively induced eye movements. $E x$ perimental Brain Research, 56, 438-447.

Brandt, T., Dichgans, J., Buchele, W. (1974). Motion habituation: Inverted self-motion perception and optokinetic after-nystagmus. Experimental Brain Research, 21, 337-352.

Brandt, T., Dichgans, J., Koenig, E. (1973). Differential effects of central versus peripheral vision on egocentric and exocentric motion perception. Experimental Brain Research, 16, 476-491.

Bridgeman, B., KLassen, H. (1983). On the origin of stroboscopic induced motion. Perception \& Psychophysics, 34, 149-154.

Brosgole, L. (1968). An analysis of induced motion. Acta Psychologica, 28, 1-44.

Carr, H. A. (1910). The autokinetic sensation. Psychological Review, 17, 42-75

Cheng, M., \& Outerbridae, J. S. (1975). Optokinetic nystagmus during selective retinal stimulation. Experimental Brain Research, 23, 129-139.

Dubols, M. F. W., \& Collewis, H. (1979). Optokinetic reactions in man elicited by localized retinal motion stimuli. Vision Research, 19. $1105-1115$.

Duncker, K. (1929). Uber induzierte Bewegung. Psychologische Forschung, 12, 180-259.

Evanoff, J. N., LACKNER, J. R. (1987). Influence of maintained ocular deviation on the spatial displacement component of the oculogyral illusion. Perception \& Psychophysics, 42, 25-28.

Fischer, M. H., KorNMüLLER, A. E. (1930). Optokinetisch ausgeloste Bewegungswahrnehmungen und optokinetischer Nystagmus. Journal fir Psychologie und Neurologie (Leipzig), 41, 273-308.

GoGeL, W. C. (1977). Independent motion induction in separate portions of the visual field. Bulletin of the Psychonomic Society, 10, 408-410.

GOGEL, W. C., \& GRIFFIN, B. W. (1982). Spatial induction of illusory motion. Perception, 11, 187-199.

GoGel, W. C., \& KosLow, M. (1972). The adjacency principle and induced movement. Perception \& Psychophysics, 11, 309-314.
Gogel, W. C., \& MacCracken, P. J. (1979). Depth adjacency and induced motion. Perceptual \& Motor Skills, 48, 343-350.

Gogel, W. C., SHARKEY, T. J. (1989). Measuring attention using induced motion. Perception, 18, 303-320.

Gogel, W. C., \& TIETZ, J. D. (1976). Adjacency and attention as determiners of perceived motion. Vision Research, 28, 681-694.

HeCKMANN, T. (1986). Induced motion and orienting responses: A new model of relationships among optokinetic afternystagmus, induced motion, egocentric orientation and vection. Doctoral dissertation, University of California, Davis.

Heckmann, T., \& Howard, I. P. (in press). Induced motion: Isolation and dissociation of egocentric and vection-entrained components. Perception.

Heckmann, T., \& Post, R. B. (1988). Induced motion and optokinetic afternystagmus: Parallel response dynamics with prolonged stimulation. Vision Research, 28, 681-694.

Holm-JENSEN, S. (1984). The significance of attention and duration of the stimulation in optokinetic nystagmus. Acta Otolaryngologica, 98, 21-29.

Howard, I. P., Giaschi, D., Murasugi, C. M. (1989). Suppression of OKN and VOR by afterimages and imaginary objects. $E x$ perimental Brain Research, 75, 139-145.

HowaRD, I. P., Gonzalez, E. G. (1987). Human optokinetic nystagmus in response to moving binocularly disparate stimuli. Vision Research, 27, 1807-1816.

HowARD, I. P., SIMPSON, W. (1989). Human optokinetic nystagmus is linked to the stereoscopic system. Experimental Brain Research, 78, 309-314.

Johansson, G. (1977). Studies in visual perception of locomotion. Perception, 6, 365-376.

LARSBY, B., HYDEN, D., \& OdQVist, L. (1984). Gain and phase characteristics of compensatory eye movements in light and darkness. Acta Otolaryngologica, 97, 223-232.

Leibowitz, H. W., ShuperT, C., Post, R. B., \& Dichgans, T. (1983). Autokinetic drifts and gaze deviation. Perception \& Psychophysics, 33, 455-459.

LEVI, D. M., SCHOR, C. M. (1984). Spatial and velocity tuning of processes underlying induced motion. Vision Research, 24, 1189-1196.

Mack, A., \& Bachant, J. (1969). Perceived movement of the afterimage during eye movements. Perception \& Psychophysics, 6 , 379-384.

NAKAYAMA, K., \& TyLER, C. W. (1978). Relative motion induced between stationary lines. Vision Research, 18, 1663-1668.

Post, R. B. (1986). Induced motion considered as a visually induced oculogyral illusion. Perception, 15, 131-138.

Post, R. B., \& Chaderjian, M. (1988). The sum of real and induced motion is not a straight path. Perception \& Psychophysics, 43, 121-124.

Post, R. B., Chi, D., Heckmann, T., \& Chaderuian, M. (1989). A reevaluation of the effect of velocity on induced motion. Perception \& Psychophysics, 45, 411-416.

Post, R. B., \& HeckmanN, T. (1986). Induced motion and apparent straight ahead during prolonged stimulation. Perception \& Psychophysics, 40, 263-270.

Post, R. B., LEIBowitz, H. W. (1982). The effect of convergence on the vestibulo-ocular reflex and implications for perceived movement. Vision Research, 22, 461-465.

Post, R. B., \& Leibowitz, H. W. (1985). A revised analysis of the role of efference in motion perception. Perception, 14, 631-643.

Post, R. B., LoTT, L. A. (1990). Relationship of induced motion and apparent straight-ahead shifts to optokinetic stimulus velocity. Perception \& Psychophysics, 48, 401-406.

REINHARDT-RUTLAND, A. H. (1988). Induced movement in the visual modality. Psychological Bulletin, 103, 57-71.

Rock, I., Auster, M., SChiffman, M., \& Wheeler, D. (1982). Induced movement based on subtraction of motion from the moving object. Journal of Experimental Psychology: Human Perception \& Performance, 6, 391-403.

Schor, C. M., Lakshminarayanan, V., Narayan, V. (1984). Optokinetic and vection responses to apparent motion in man. Vision Research, 24, 1181-1187. 
Steinbach, M. J. (1976). Pursuing the perceptual rather than the retinal stimulus. Vision Research, 16, 1371-1376.

WadE, N. J., \& Swanston, M. T. (1987). The representation of nonuniform motion: Induced motion. Perception, 16, 555-571.

Wallach, H., Bacon, J., Schulman, P. (1978). Adaptation in motion perception: Alteration of induced motion. Perception \& Psychophysics, 24, 509-514.

WaLlach, H., BeCKLEN, R. (1983). An effect of speed on induced motion. Perception \& Psychophysics, 34, 237-242.

WARren, H. C. (1895). Sensations of rotation. Psychological Review, 2, 273-276.
WhitTAKer, S. G., EAHOLtz, G. (1982). Learning patterns of eye motion for foveal pursuit. Investigative Ophthalmology \& Visual Science, 23, 393-397.

WyATT, H. J., \& PolA, J. (1979). The role of perceived motion in smooth pursuit eye movements. Vision Research, 19, 613-618.

WyatT, H. J., \& PoLA, J. (1984). A mechanism for suppression of optokinesis. Vision Research, 24, 1931-1945.

(Manuscript received February 7, 1990; revision accepted for publication May 6, 1991.) 